\title{
Improved hatchability and efficient protection after in ovo vaccination with live-attenuated H7N2 and H9N2 avian influenza viruses
}

Yibin Cai ${ }^{1,2}$, Haichen Song ${ }^{3}$, Jianqiang Ye ${ }^{1,2}$, Hongxia Shao ${ }^{1,2}$, Rangarajan Padmanabhan ${ }^{1,2,4}$, Troy C Sutton ${ }^{1,2}$, Daniel R Perez ${ }^{1,2^{*}}$

\begin{abstract}
Mass in ovo vaccination with live attenuated viruses is widely used in the poultry industry to protect against various infectious diseases. The worldwide outbreaks of low pathogenic and highly pathogenic avian influenza highlight the pressing need for the development of similar mass vaccination strategies against avian influenza viruses. We have previously shown that a genetically modified live attenuated avian influenza virus (LAIV) was amenable for in ovo vaccination and provided optimal protection against H5 HPAl viruses. However, in ovo vaccination against other subtypes resulted in poor hatchability and, therefore, seemed impractical. In this study, we modified the $\mathrm{H} 7$ and $\mathrm{H} 9$ hemagglutinin (HA) proteins by substituting the amino acids at the cleavage site for those found in the H6 HA subtype. We found that with this modification, a single dose in ovo vaccination of 18day old eggs provided complete protection against homologous challenge with low pathogenic virus in $\geq 70 \%$ of chickens at 2 or 6 weeks post-hatching. Further, inoculation of 19-day old egg embryos with $10^{6}$ EID $_{50}$ of LAIVs improved hatchability to $\geq 90 \%$ (equivalent to unvaccinated controls) with similar levels of protection. Our findings indicate that the strategy of modifying the HA cleavage site combined with the LAIV backbone could be used for in ovo vaccination against avian influenza. Importantly, with protection conferred as early as 2 weeks post-hatching, with this strategy birds would be protected prior to or at the time of delivery to a farm or commercial operation.
\end{abstract}

\section{Introduction}

Although depopulation of infected flocks is the method of choice to control the spread of avian Influenza virus (AIV) in poultry, vaccination has become an alternative strategy in order to provide protection to high-risk birds and reduce the possibility of transmission among birds and/or to mammals $[1,2]$. Thus, in many countries in which avian influenza outbreaks particularly of low pathogenicity have occurred recurrently, selective culling followed by vaccination is used as a measure to control the disease without major economic disruptions. There are only two types of avian influenza vaccines (AIVs) licensed worldwide: inactivated whole AIV vaccine and recombinant fowlpox virus-vectored vaccine expressing the HA gene of AIV. However, both types of vaccines

\footnotetext{
* Correspondence: dperez1@umd.edu

'Department of Veterinary Medicine, University of Maryland, College Park,

8075 Greenmead Drive, College Park, MD 20742-3711, USA

Full list of author information is available at the end of the article
}

have major limitations: inactivated vaccines cannot elicit strong mucosal and cellular immunity; and previous exposure to fowlpox virus inhibits the host response to the fowl-pox vectored vaccine inhibiting anti-influenza immunity [2-4]. In addition, both strategies are heavily time-consuming, requiring each bird to be vaccinated individually by parenteral inoculation.

With the advent of reverse genetics, LAIVs have emerged as a potential alternative to control avian influenza [5]. Several different strategies have been developed to attenuate influenza viruses based on mutations in one or more of the viral internal or surface genes [6-9]. Several studies have shown that LAIV vaccines protect against influenza viruses of low or high pathogenicity in poultry and mammals. However, field application of these vaccines is difficult due to the inherent segmented nature of the influenza genome and the fear that LAIVs could expand the plethora of influenza viruses through reassortment. Despite recent reports of the potential
Ciomed Central

C 2011 Cai et al; licensee BioMed Central Ltd. This is an Open Access article distributed under the terms of the Creative Commons Attribution License (http://creativecommons.org/licenses/by/2.0), which permits unrestricted use, distribution, and reproduction in any medium, provided the original work is properly cited. 
genomic manipulation of influenza to prevent undesired reassortments, it is unclear how these viruses will behave under more natural conditions; either by providing adequate protection or reverting to wild type-like viruses. Instead, in ovo vaccination using LAIV is an attractive alternative to provide fast and effective protection against influenza while avoiding the potential for reassortment (in ovo vaccination is unlikely to produce reassortants as other influenza viruses are not present in the egg).

Several strategies have been developed to generate LAIVs for in ovo vaccination. A recombinant LAIV was recently developed that provided immunity against HPAI H5N1 influenza and Newcastle Disease Virus (NDV) $[7,10]$. This recombinant influenza virus expressed the $\mathrm{HA}$ of $\mathrm{H} 5$ with a deleted polybasic cleavage site, and the ectodomain of the hemagglutinin-neuraminidase (HN) genes NDV instead of NA gene of HPAI H5N1. With this bivalent virus, a single dose in ovo vaccination of 18 day-old eggs provided $90 \%$ and $80 \%$ protection as early as 3 weeks post-hatching, against NDV and HPAI, respectively. A second strategy employed a non-replicating human adenovirus serotype 5 (Ad5)- vectored vaccine that expressed the HA of a LPAI H5N9 virus. Similarly, this vaccine was delivered in ovo and conferred protection in chickens after challenge with either HPAI H5N1 (89\% HA homology; $68 \%$ protection) or HPAI H5N2 (94\% HA homology; $100 \%$ protection) viruses. Unfortunately, in both these studies, the hatchability efficiency was not addressed in detail [11].

In our previous reports we demonstrated the potential of a genetically modified LAIV with the internal gene backbone of A/guinea fowl/Hong Kong/WF10/99 (H9N2) (WF10att) as a vaccine backbone for H5N1 influenza viruses [2]. The WF10att backbone carries mutations in the PB1 (K391E, E581G and A661T) and PB2 (N265S) genes. In addition an HA tag was cloned in frame at the C-terminus of PB1, and enhanced the att phenotype. This backbone results in virus attenuation in vitro while attaining high viral growth properties at the permissive temperatures of 33 and $35^{\circ} \mathrm{C}$. We also showed that an $\mathrm{H} 5 \mathrm{~N} 1$ virus carrying the backbone $\Delta \mathrm{H} 5 \mathrm{~N} 1 \mathrm{WF} 10$ att was amenable for in ovo vaccination and provided optimal protection against H5 HPAI virus. More specifically, a single low $\left(10^{4}\right.$ EID $\left._{50}\right)$ or high $\left(10^{6}\right.$ EID $_{50}$ ) dose of LAIV resulted in greater than $60 \%$ protection at 4-week post-hatching and $100 \%$ protection at 9 to 12 -week post-hatching. Incorporation of a boost regime with either the low or high virus dose at 2-weeks post-hatching increased the protection efficiency to $100 \%$ in 4-week old chickens. The hatchability efficiency of the high-dose $\left(10^{6}\right.$ EID $\left._{50}\right)$ in ovo vaccination was $85 \%$, compared with $90 \%$ in low-dose $\left(10^{4}\right.$ EID $\left._{50}\right)$ and mock groups $[2,12]$.
In ovo vaccination with live attenuated viruses is widely used in commercial poultry against various infectious diseases. In ovo vaccination was initially introduced into the poultry market to protect against Marek's disease virus (MD) $[13,14]$. Currently, over $80 \%$ of US broilers are immunized in ovo with $\mathrm{MD}$ vaccine. In ovo vaccination is also effective and used commercially to protect poultry from infectious bursal disease virus (IBDV) [15]. Compared with field vaccination, in ovo vaccination provides uniform and fast delivery $(50,000$ egg/h), reduced labor costs, decreased stress to the birds; and most importantly, elicits early immune responses, as soon as 2 -week post hatching [16]. From practical and commercial perspectives, in ovo vaccination not only has to be effective in providing protection but also has to maintain high hatchability levels $(\geq 90 \%)$. In this report, we investigated the effects of changing the $\mathrm{H} 7$ and $\mathrm{H} 9$ cleavage site to that of the LPAI H6 subtype and the timing of vaccination on levels of protection and hatchability after in ovo vaccination with LAIV against $\mathrm{H} 7$ and H9 LPAI viruses. Our results indicate that in ovo vaccination can result in significant protection against the $\mathrm{H} 7$ and $\mathrm{H} 9$ virus subtypes while maintaining high hatchability $(>90 \%)$ when the vaccine is administered in 19-day old chicken embryos.

\section{Materials and methods}

Viruses, cells and animals

The influenza virus A/Guinea Fowl/Hong Kong/WF10/ 99 (H9N2) (WF10) was kindly provided by Robert Webster from the repository at St. Jude's Children's Research Hospital, Memphis, Tennessee; influenza virus A/Chicken/Delaware/VIVA/04 (H7N2) (CK/04) was kindly obtained from Dennis Senne at the National Veterinary Laboratory Services, USDA, Ames, Iowa. The viruses were propagated in 10-day-old embryonated specific-pathogen-free chicken eggs at $35^{\circ} \mathrm{C}$ and stored at $-70^{\circ} \mathrm{C}$. The viruses were titrated by the Reed and Muench method to determine the $50 \%$ egg infectious dose $\left(\right.$ EID $\left._{50}\right)$ [17]. 293T human embryonic kidney and Madin-Darby canine kidney (MDCK) cells were maintained as described previously [2]. White leghorn chickens (Charles River Laboratories, MA) and Japanese quail (Murray McMurray Hatchery, Webster, IA) were hatched at $100^{\circ} \mathrm{F}$ in a circulating air incubator (G.Q.F. Manufacturing co. Savannah, GA) and maintained under BSL2 conditions.

\section{Generation of recombinant virus by reverse genetics}

The 6 internal genes of WF10att were described previously and were used to recover viruses carrying the surface genes of Ck/04 or WF10 [2]. The cloning of the $\mathrm{Ck} / 04$ surface genes has been previously described [2]. The H7 HA cleavage site, PEKPKPRG, was substituted 
with an alternative cleavage site sequence, PQIETRG, from the H6 HA subtype using a two-step PCR reaction and the plasmid pDP2002-H7 $(\mathrm{Ck} / 04)$ as the template (Figure 1A). In brief, two PCR fragments were produced by using primers EcoR I 550-F (5'-CTGTCGAATTCAGATAATTCAGC-3') and H7-H6 CVS-R (5'-GGTCTCCCGCTGTGGAACATTTCTC-3'), and primers H7-H6 CVS-F (5'-CACAGCGGGAGACCAGAGGCCTTTTTG$\left.3^{\prime}\right)$ and Pst I 1150-R (5'-GTCAGCTGCAGTTCCCTCCCCTTGT-3'). These two fragments were then used as templates for a new PCR product using primers EcoR I 550-F and Pst I 1150-R. The fragment was digested with EcoR I and Pst I, and cloned into pDP-2002-H7 (VIVA/04), to obtain pDP2002-mH7.

The H9 HA cleavage site, PARSSRG, was substituted with the alternative cleavage site sequence PQIETRG (Figure 1B) using pDPH9WF10 as the template. Two PCR fragments were produced by using primers: Xbal I 285-F (5'-CCTCATTCTAGACACATGCAC-3') and H9H6 CVS-R (5'-CCAAATAGTCCTCTAGTTTCGATCT GAGGCACGTTC-3'), and primers H9-H6 CVS-F (5'GAACGTGCCTCAGATCGAAACTAGAGGACTATT TGG-3') and EcoN I 1297-R (5'-CCTCATTCTAGACA CATGCAC-3'). These two fragments were then used as templates to generate a new PCR fragment using primers Xbal I 285-F and EcoN I 1297-R. The fragment was digested with Xbal I and EcoN I, and cloned into pDPH9WF10, resulting in the formation of pDP-2002mH9.

Recombinant viruses were generated using the 8 plasmid system in co-cultured 293T and MDCK cells as described previously [2]. The recombinant viruses (Table 1) were propagated in 10-day-old embryonated eggs, titrated by $\mathrm{EID}_{50}$, and stored at $-70^{\circ} \mathrm{C}$ until use. 2mH7N2:6WF10att and 2mH9N2:6WF10att viruses were sequenced using specific primers, the Big Dye Terminator v3.1 Cycle Sequencing kit (Applied Biosystems,

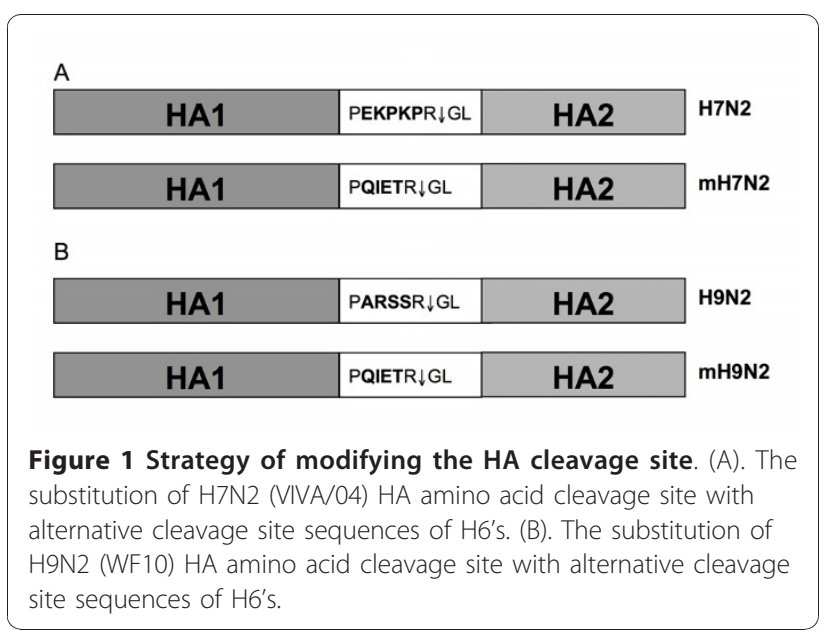

Table 1 Gene constellations of recombinant viruses used in this study

\begin{tabular}{cccc}
\hline Virus & HA & NA & $\begin{array}{c}\text { Internal genes } \\
\text { (PB1, PB2, PA, NP, } \\
\text { M and NS) }\end{array}$ \\
\hline 2m2H7N2:6WF10att & mH7 (VIVA/04) & N2 (VIVA/04) & WF10att \\
\hline 2H7N2:6WF10att & H7 (VIVA/04) & N2 (VIVA/04) & WF10att \\
\hline 2mH9N2:6WF10att & mH9 (WF10) & N2 (WF10) & WF10att \\
\hline 2H9N2:6WF10att & H9 (WF10) & N2 (WF10) & WF10att \\
\hline
\end{tabular}

Foster City, CA), and a 3100 Genetic Analyzer (Applied Biosystems, Foster City, CA), according to the manufacturer's instructions. The genetic stability of mutations on HA, PB1 and PB2 were evaluated by serial passage of virus stocks at a 1:10,000 dilution for 10 passages in triplicate samples in 10-day-old embryonated eggs. Viruses obtained after ten passages were sequenced as described above.

\section{Hatchability in embryonated chicken eggs}

18 or 19-day-old embryonated specific-pathogen-free chicken eggs were inoculated with either $10^{6}$ or $10^{7}$ EID $_{50}$ of virus in $0.1 \mathrm{ml}$ inoculum according to the scheme presented in Table 2. Eggs in the mock group were inoculated with $0.1 \mathrm{ml}$ of PBS. The egg inoculation was performed as described previously [2]. Briefly, eggs were candled, and a small hole was made through the air cell with an electric drill. Next, $0.1 \mathrm{ml}$ of virus dilution or PBS was injected into the allantoic cavity using a 21 -gauge needle at a depth of $2.5 \mathrm{~cm}$. The percent hatchability was calculated using the total number of inoculated eggs versus the number of 21-day old eggs that hatched in each group. This experiment was performed under BSL-2 conditions according to protocols approved by the Animal Care and Use Committee of the University of Maryland.

\section{Plaque assay in chicken embryonic kidney (CEK) cells and immunostaining}

To investigate if the replacement of amino acids at the HA cleavage site affected the temperature sensitive phenotype of the new live-attenuated viruses, plaque assays were performed in CEK cells at $37^{\circ} \mathrm{C}, 39^{\circ} \mathrm{C}$, and $41^{\circ} \mathrm{C}$. Confluent CEK cell monolayers in six-well plates were infected with $0.5 \mathrm{ml}$ of 10 -fold dilutions of virus 2mH7N2:6WF10att or 2H7N2:6WF10att in M199 medium. The cells were incubated with the virus dilutions for $1 \mathrm{~h}$ at $37^{\circ} \mathrm{C}$, washed, and overlaid with M199 medium containing $0.9 \%$ agar and $0.1 \mu \mathrm{g} / \mathrm{ml}$ TPCK-trypsin. The plates were then incubated at $37^{\circ} \mathrm{C}, 39^{\circ} \mathrm{C}$, and $41^{\circ} \mathrm{C}$ with $5 \% \mathrm{CO}_{2}$. At 4 days post-inoculation (dpi) the overlay was removed and immunostaining was performed as described previously [2]. In brief, the cells were fixed, 
Table 2 Comparison of the hatchability of new recombinant viruses in embryonated chicken eggs vs. the viruses with wild type HAs and the optimization of the dose and timing for in-ovo vaccination

\begin{tabular}{|c|c|c|c|c|}
\hline \multicolumn{2}{|c|}{ Vaccine } & \multirow{2}{*}{$\frac{\text { Dose (EID50) }}{10^{6}}$} & \multirow{2}{*}{$\frac{\text { Embryo age (Day) }}{18}$} & \multirow{2}{*}{$\frac{\text { \% Hatchability (\# hatched/total \#) }}{50 \%(15 / 30)}$} \\
\hline H7N2 (VIVA/04) Vaccine & 2mH7N2:6WF10 att & & & \\
\hline & & & & $(P=0.016)$ \\
\hline & & $10^{6}$ & 19 & $93 \%(42 / 45)$ \\
\hline & & & & $(P=0.061)$ \\
\hline & & $10^{7}$ & 19 & $80 \%(24 / 30)$ \\
\hline & & & & $(P=0.066)$ \\
\hline & 2H7N2:6WF10att & $10^{6}$ & 18 & $30 \%(9 / 30)$ \\
\hline & & $10^{6}$ & 19 & $43 \%(13 / 30)$ \\
\hline & & $10^{7}$ & 19 & $37 \%(11 / 30)$ \\
\hline \multirow[t]{9}{*}{ H9N2 (WF10) Vaccine } & 2mH9N2:6WF10att & $10^{6}$ & 18 & $63 \%(19 / 30)$ \\
\hline & & & & $(P=0.0161)$ \\
\hline & & $10^{6}$ & 19 & $90 \%(27 / 30)$ \\
\hline & & & & $(P=0.260)$ \\
\hline & & $10^{7}$ & 19 & $83 \%(25 / 30)$ \\
\hline & & & & $(P=0.154)$ \\
\hline & 2H9N2:6WF10att & $10^{6}$ & 18 & $37 \%(11 / 30)$ \\
\hline & & $10^{6}$ & 19 & $60 \%(18 / 30)$ \\
\hline & & $10^{7}$ & 19 & $37 \%(11 / 30)$ \\
\hline \multirow[t]{2}{*}{ PBS (Mock) } & & 0 & 18 & $93 \%(28 / 30)$ \\
\hline & & 0 & 19 & $96 \%(43 / 45)$ \\
\hline
\end{tabular}

* 3 chickens dead at 2-5 days post-hatching.

permeabilized, and blocked with bovine serum albumin (BSA) in PBS. The cells were then incubated with mouse anti-WF10 monoclonal NP antibody prepared in our laboratory, followed by incubation with peroxidaseconjugated goat anti-mouse IgG (Jackson Immuno Research, West Grove, PA). The presence of viral antigen was revealed by adding several drops of aminoethylcarbazol (BD Biosciences, San Diego, CA). The size and number of plaques at each temperature were compared to determine the temperature sensitive phenotype of the new recombinant virus.

\section{Viral replication in MDCK cells}

Viral replication was studied to examine the temperature sensitive phenotype of the new recombinant viruses in MDCK cells. Confluent monolayers of MDCK cells in 6-well plates were infected with $2 \mathrm{~m} 2 \mathrm{H} 7 \mathrm{~N} 2: 6 \mathrm{WF} 10 \mathrm{att}$ or 2H7N2:6WF10att at a MOI $=0.001$ and cultured at $35^{\circ}$ $\mathrm{C}$ and $39^{\circ} \mathrm{C}$, respectively. Supernatant samples were collected at 12, 24, 48, 72, 96 and $120 \mathrm{~h}$ post-inoculation, and the viral titer of these samples was determined by $\mathrm{TCID}_{50}$ in MDCK cells [2].

\section{Virus replication and transmission in quail}

To evaluate the vaccine's attenuated phenotype in vivo, 2mH7N2:6WF10att was compared to the recombinant virus 2H7N2:6WF10att. Six 4-week-old Japanese quail were inoculated by the ocular, intranasal, and intratracheal routes with $10^{6}$ EID $_{50} / 0.5 \mathrm{ml}$ of either 2mH7N2:6WF10att or 2H7N2:6WF10att vaccine viruses. Two control quail were inoculated with $0.5 \mathrm{ml}$ of PBS. At 1 dpi, 3 naïe quail were introduced into the same isolators, and placed in direct contact with the inoculated quail to assess virus transmission. At $3 \mathrm{dpi}$, 3 inoculated quail per group were sacrificed, lungs were homogenized and virus titers were determined by EID $_{50}$. For the remaining quail, tracheal and cloacal swabs were collected from both the inoculated and direct contact birds at 1, 3, 5, 7, and 9 dpi. The swab samples were stored in glass vials in $1.0 \mathrm{ml}$ freezing Brain Heart Infusion (BHI) medium (BD, Sparks, MD) and titrated for infectivity in 10-day-old embryonated chicken eggs and MDCK cells. Sera were collected 2 weeks post-infection and HA inhibition tests (HI) were performed to quantify antibodies against HA [18].

\section{Challenge studies}

Chickens that hatched after in ovo vaccination were randomly divided into two groups with the same number of individuals. Early protection was assessed in the first group of chickens by challenge at 2-weeks post-hatching. Challenge virus consisted of $5 \times 10^{5} \mathrm{EID}_{50}$ of virus (equal to 500 chicken infectious dose $\left.50\left(\mathrm{CID}_{50}\right)\right)$ and was delivered via intranasal inoculation. Late protection 
was assessed in the second group of chickens following the strategy described above, but in chickens that were 6 weeks old. Tracheal and cloacal swab samples were collected at 3, 5, and 7 days post-challenge (dpc). Virus shedding was titrated in MDCK cells by $\mathrm{TCID}_{50}$. Sera samples were collected at 2 -weeks post-hatching prechallenge, and 2 weeks post-challenge. HI titers were determined as previously described [18]. Animal studies were conducted under BSL-2 conditions, and performed according to protocols approved by the Animal Care and Use Committee of the University of Maryland.

\section{Results}

Chicken hatchability is impaired after in ovo vaccination with H7N2 and H9N2 WF10att viruses

Our previous studies showed that in ovo vaccination with $10^{6} \mathrm{EID}_{50}$ of the $\Delta \mathrm{H} 5 \mathrm{~N} 1: 6 \mathrm{WF} 10$ att virus resulted in effective protection against HPAI H5N1 virus [2]. We wanted to determine whether similar levels of protection could be obtained against other HA subtypes following the same strategy. We were particularly interested in the $\mathrm{H} 7$ and the $\mathrm{H} 9$ subtypes because they have been responsible for recurrent outbreaks, particularly in Eurasia (although in our studies a $\mathrm{H} 7$ virus of the North American lineage was used). Thus, 18-day-old egg embryos were inoculated with $10^{6} \mathrm{EID}_{50}$ of either 2H7N2:6WF10att or 2H9N2:6WF10att vaccine viruses (Tables 1 and 2). Unfortunately, the hatchability of vaccinated eggs was poor, $30 \%$ and $37 \%$ in eggs vaccinated with $2 \mathrm{H} 7 \mathrm{~N} 2: 6 \mathrm{WF} 10 \mathrm{att}$ and 2H9N2:6WF10att, respectively (Table 2 ) compared to $85 \%$ in eggs vaccinated with the $2 \Delta \mathrm{H} 5 \mathrm{~N} 1: 6 \mathrm{WF} 10$ att virus (not shown and [2]).

\section{Chicken hatchability after modification of the HA cleavage site in H7N2 and H9N2 WF10att viruses}

The 2 $\Delta \mathrm{H} 5 \mathrm{~N} 1: 6 \mathrm{WF} 10 \mathrm{att}$ virus carries the H5 HA protein from A/Vietnam/1203/04 (H5N1) but its polybasic cleavage site, characteristic of HPAI viruses, has been replaced with that from the LPAI H6 HA virus subtype, as described in previous reports [19]. In order to determine if incorporation of the H6 HA cleavage site in the $\mathrm{H} 7$ and $\mathrm{H} 9$ subtypes would result in more attenuated vaccine viruses and improved hatchability, we generated the recombinant viruses 2mH7N2:6WF10att and 2mH9N2:6WF10att. Modifications at the cleavage site in these viruses did not have major effects on the in vitro properties of these viruses. Both recombinant viruses reached titers of $10^{6} \mathrm{TCID}_{50} / \mathrm{ml}$ at $120 \mathrm{~h}$ postinfection in MDCK cells inoculated at an $\mathrm{MOI}=0.001$ and cultured at $35^{\circ} \mathrm{C}$ (Figure 2 and data not shown). In contrast, viral replication at $39^{\circ} \mathrm{C}$ was severely restricted, with viral titers reduced more than 1000 -fold relative to those at $35^{\circ} \mathrm{C}$ (Figure 2 and data not shown). This indicates that modifications in the HA cleavage site did not change the temperature sensitive phenotype of these

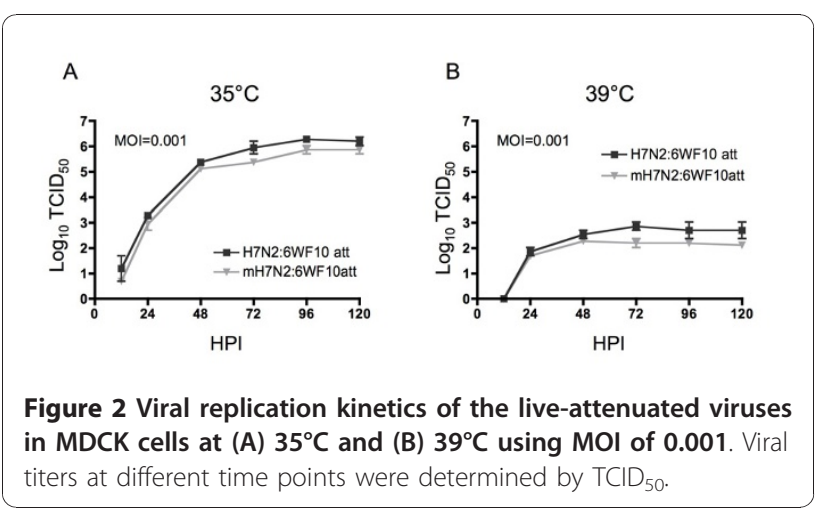

viruses in MDCK cells. Likewise, plaque assays, performed using CEK cells (Figure 3), showed that 2mH7N2:6WF10att formed significantly smaller plaques than $2 \mathrm{H} 7 \mathrm{~N} 2: 6 \mathrm{WF} 10$ att at $37^{\circ}$ and $39^{\circ} \mathrm{C}$. As expected, these viruses were highly restricted at $41^{\circ} \mathrm{C}$ (yields of $<10^{3} \mathrm{PFU} / \mathrm{ml}$ ) consistent with their att phenotype. Interestingly, the lower virus titers and smaller plaque sizes of 2mH7N2:6WF10att compared to 2H7N2:6WF10att indicate an additive effect on attenuation provided by the modified HA cleavage site. Similar results were obtained when we compared the 2mH9N2:6WF10att to 2H9N2:6WF10att (not shown). However, despite the additional attenuation, only a slight improvement in hatchability (50\% and 63\%) was observed when 18-dayold egg embryos were inoculated with $10^{6} \mathrm{EID}_{50}$ of the 2mH7N2:6WF10att and 2mH9N2:6WF10att vaccine viruses, respectively (Table 2 ).

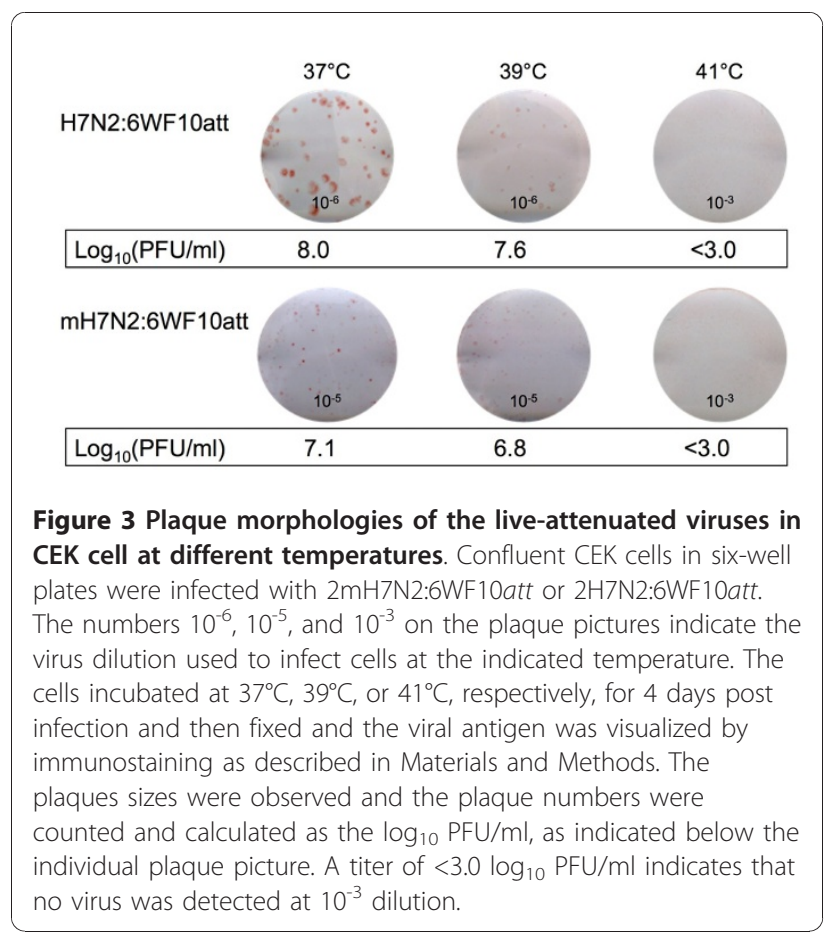


Chicken hatchability is improved when in ovo vaccination is performed on 19-day old chicken embryos

The previous hatchability results suggested that additional mutations in the virus genome were required or that the conditions under which the vaccine was delivered needed to be changed to improve hatchability. Certainly, additional mutations in the viral genome could be introduced, however, they might also affect immunogenicity.

Thus, we chose to deliver the vaccine to 19-day old chicken embryos and compare hatchability to vaccination of 18-day old chicken embryos. In ovo vaccination of 19-day old chicken embryos was performed with either $10^{6}$ or $10^{7} \mathrm{EID}_{50}$ to explore hatchability efficiency with two different virus concentrations. Interestingly, hatchability was greatly improved in 19-day-old vaccinated embryos. Hatchability reached $93 \%$ and $90 \%$ in the 2mH7N2:6WF10att and 2mH9N2:6WF10att groups, respectively, when eggs were vaccinated with $10^{6} \mathrm{EID}_{50}$ (Table 2). As shown in Table 2, an increase in virus delivery dose to $10^{7} \mathrm{EID}_{50}$ was detrimental for hatching. These results suggest that in ovo vaccination in 19-day old chicken embryos may be a suitable strategy to generate an anti-influenza response in chickens.

\section{Modification of the HA cleavage site reduces replication of $2 \mathrm{mH7N2}$ :6WF10att virus in quail}

We have previously shown that quail are more susceptible than chickens to avian influenza viruses. Thus quail represent a better host to test whether modifications in our vaccine viruses would have any effect on replication and transmissibility. To investigate if modification of the HA cleavage site altered the degree of attenuation and transmissibility in quail, 2 groups of quail $(n=6)$ were inoculated with either the $2 \mathrm{mH} 7 \mathrm{~N} 2: 6 \mathrm{WF} 10$ att virus or the 2H7N2: 6WF10att virus. At 24 h after infection, 3-naïve quail/group were brought in direct contact with inoculated quail to monitor for transmission (Table 3). At $3 \mathrm{dpi}, 3$ inoculated quail from each group were sacrificed to determine virus load in the lungs. No virus was detected in the lungs of inoculated quail regardless of the virus used. This finding is consistent with our previous study showing that the WF10att backbone prevents the virus from replicating in the lower respiratory tract (not shown and $[2,12]$ ). In addition, no virus was detected in cloacal swabs for any of the quail in the study (not shown). In contrast, tracheal swabs showed the presence of virus in the 2H7N2:6WF10att group, with peak virus titers of $10^{2.9}$ (at $1 \mathrm{dpi}$ ) and $10^{1.6}$ $\mathrm{TCID}_{50} / \mathrm{ml}$ (at $3 \mathrm{dpi}$ ) in the inoculated and direct contact quail, respectively. Inoculated quail remained positive until 5 dpi but were negative by $7 \mathrm{dpi}$. Only 2 out of the 3 direct contact quail showed trace amounts of 2H7N2:6WF10att and were negative by 9 dpi. With respect to the 2mH7N2: 6WF10att inoculated group, only trace amounts of virus were observed, and just 1 of 3 quail remained positive by $7 \mathrm{dpi}$ and it became negative by 9 dpi. Direct contacts in the 2mH7N2: 6WF10att virus group were negative except for trace amounts of virus on a single day, $7 \mathrm{dpi}$, in 2 of the 3 quail. The levels of virus replication in the different groups corresponded with the levels of seroconversion observed. Thus, inoculated quail in the 2H7N2:6WF10att group had the highest neutralizing antibody response, followed by inoculated quail in the 2mH7N2: 6WF10att group, whereas the direct contacts in the 2H7N2:6WF10att showed low, but significant seroconversion. Also consistent with the transient presence of the $2 \mathrm{mH} 7 \mathrm{~N} 2$ : 6 WF10att virus in the direct contact group, very low seroconversion was observed. These studies suggest that alterations in the HA cleavage site have an effect on replication in vivo further attenuating these viruses and limiting the ability to replicate after transmission (Table 3). We did not perform similar studies in quail with the H9N2 vaccine viruses. However, we must note that similar studies in white leghorn chickens did not result in detectable transmission, when the viruses carry the att backbone in the context of H7N2 or H9N2 surface genes (not shown).

\section{Stability of new recombinant viruses}

The genetic stability of the mutations on HA, PB1, and PB2, was verified by serial passage of the 2mH7N2:6WF10att and 2mH9N2:6WF10att viruses in 10-day-old embryonated eggs. Amino acids 391E, 581G, $661 \mathrm{~T}$ and the HA tag on PB1, and $265 \mathrm{~S}$ on PB2 remained unchanged after serial propagation in eggs. More importantly, the amino acids at the HA cleavage site remained unchanged and corresponded to the $\mathrm{H} 6$ HA cleavage sequence (PQIETRG).

\section{Single dose in ovo vaccination provides protection in chickens from homologous challenge with $\mathrm{H} 7$ and $\mathrm{H} 9$ LPAI viruses at 2 and 6 weeks post-hatching}

To further evaluate whether in ovo immunization would result in protection against $\mathrm{H} 7$ or $\mathrm{H} 9$ viruses, vaccinated chickens were divided into two groups, and subsequently challenged with homologous virus at either 2 or 6 weeks post-hatching (Tables 4 and 5).

Pre-challenge sera collected at 2 weeks post-hatching showed limited seroconversion in chickens that received the 2mH7N2:6WF10att (Table 4), both in terms of the number of seropositive chickens as well as the level of $\mathrm{HI}$ responses. However, sera collected at 6 weeks posthatching showed increased numbers of seropositive chickens and increased HI titers (Table 4). Relative to 2mH7N2:6WF10att, improved and more consistent antibody responses were obtained in chickens that were vaccinated with 2mH9N2:6WF10att (Table 5). In terms of 
Table 3 Replication and transmission study of recombinant virus 2H7N2:6WF10att and 2mH7N2:6WF10att in quail

\begin{tabular}{|c|c|c|c|c|c|c|c|}
\hline \multirow[t]{2}{*}{ Virus } & \multirow[t]{2}{*}{ Group } & \multicolumn{5}{|c|}{$\begin{array}{l}\text { \# of positive tracheal swab/total \# post-inoculation } \\
\left(\log _{10} \mathrm{TCID}_{50} / \mathrm{ml} \pm \mathrm{SD}\right) \text { at peak viral shedding }\end{array}$} & \multirow[t]{2}{*}{$\begin{array}{l}\text { \# of seroconverted/total \# } \\
\text { (Average } \mathrm{HI} \text { titer at } 14 \mathrm{dpi}\end{array}$} \\
\hline & & $1 \mathrm{dpi}$ & $3 \mathrm{dpi}$ & $5 \mathrm{dpi}$ & $7 \mathrm{dpi}$ & $9 \mathrm{dpi}$ & \\
\hline \multirow[t]{2}{*}{ 2H7N2:6WF10att } & Inoculated & $6 / 6(2.9 \pm 0.4)$ & $6 / 6^{*}$ & $3 / 3$ & $0 / 3$ & $0 / 3$ & $3 / 3(133)$ \\
\hline & Contact & NA & $3 / 3(1.6 \pm 1.4)$ & $3 / 3$ & $2 / 3$ & $0 / 3$ & $3 / 3(47)$ \\
\hline \multirow[t]{2}{*}{ 2mH7N2:6WF10att } & Inoculated & $6 / 6(<0.7)$ & $6 / 6^{*}$ & $1 / 3$ & $1 / 3$ & $0 / 3$ & $3 / 3(87)$ \\
\hline & Contact & NA & $0 / 3$ & $0 / 3$ & $2 / 3(<0.7)$ & $0 / 3$ & $2 / 3(10)$ \\
\hline
\end{tabular}

* 3 quail from each inoculated group were sacrificed at 3 dpi to determine virus load in the lungs.

protection, significant protection was observed in chickens challenged with $500 \mathrm{CID}_{50}$ of $\mathrm{Ck} / 04(\mathrm{H} 7 \mathrm{~N} 2)$ at 2 or 6 weeks post-hatching but only in the 19-day old embryo vaccinated groups. Tracheal virus shedding was detected in only 2 out 8 and 1 out of 5 chickens in the 19-day old embryo groups that received $10^{6}$ or $10^{7}$ EID $_{50}$, respectively, of $2 \mathrm{mH} 7 \mathrm{N2}$ :6WF10att. There was also a sharp decrease in cloacal virus shedding in these groups, with just 1 out $8\left(10^{6}\right.$ EID $_{50}$ group $)$ and 1 out $5\left(10^{7} \mathrm{EID}_{50}\right.$ group) virus positive chickens and only at $7 \mathrm{dpc}$ (Table 4). In contrast, in the 18-day old embryo vaccinated group only 1 out 4 and 2 out 4 , at 2 and 6 weeks post-hatching, respectively, showed protection and no detectable virus replication. Similar protective responses were observed in the WF10(H9N2) challenged chickens. Chickens in the 19-day old embryo vaccinated groups showing the best protection, and those in the 18-day old embryo vaccinated groups showed the decreased protection (Table 5). Significant seroconversion in all the groups at $14 \mathrm{dpc}$ indicated that lack of virus shedding in protected chickens was not due to a failure in our challenge approach. Considering the $10^{6}$ EID $_{50}$ vaccine dose in the 19-day old embryo vaccinated groups for both att vaccines, there was between 70 and $80 \%$ protection efficiency in chickens challenge at 2 or
6 weeks post-hatching, respectively. Slightly better protection efficiency $(82 \%)$ was observed in the $10^{7}$ EID $_{50}$ vaccine dose groups; however, it was achieved at the expense of lower hatchability rates $\left(\sim 91 \%\right.$ for the $10^{6}$ EID $_{50}$ versus $\sim 80 \%$ for the $10^{7}$ EID $_{50}$ groups). In contrast, an average of only $55 \%$ protection efficiency was observed in the groups vaccinated with a dose $10^{6}$ EID $_{50}$ in 18-day old embryos.

\section{Discussion}

The HA is perhaps the most important protein in influenza viruses, as it is a critical determinant of host range and virulence $[20,21]$. The HA protein, encoded in segment 4 , is expressed on the virus surface as homotrimers. It is initially produced as a precursor, HA0, that requires post-translational modifications, including cleavage and glycosylation in order to become fully active [22]. Cleavage of the HA0 precursor leads to two subunits, HA1 - N-proximal - and HA2 - C-proximal -, which are maintained covalently linked via disulfide bonds. Trypsin-like host proteases found in the lumen of the respiratory and intestinal tracts are involved in the cleavage of the HA of low pathogenic avian influenza viruses - LPAIV - (and mammalian influenza viruses) [22]. Intracellular furin-like proteases have been

Table 4 Single-dose 2mH7N2:6WF10att in-ovo vaccination study in chickens challenged with low-pathogenic H7N2 (Ck/04) at 2 and 6 weeks post-hatching

\begin{tabular}{|c|c|c|c|c|c|c|c|c|c|}
\hline \multirow{3}{*}{$\begin{array}{c}\text { Vaccine } \\
\text { dose }\left(\text { EID }_{50}\right) / \\
\text { embryo } \\
\text { age (days) }\end{array}$} & \multirow{3}{*}{$\begin{array}{c}\text { \# positive } \\
\text { HI/total \# } \\
\text { pre-challenge } \\
\text { (HI titer) }\end{array}$} & \multirow{3}{*}{$\begin{array}{l}\text { Age } \\
\text { (in weeks) } \\
\text { at time of } \\
\text { challenge }\end{array}$} & \multicolumn{6}{|c|}{$\begin{array}{l}\text { \# Shedding virus/total \# in swabs } \\
\quad\left(\log _{10} \mathrm{TCID}_{50} / \mathrm{ml} \pm \mathrm{SD}\right)\end{array}$} & \multirow{3}{*}{$\begin{array}{c}\text { \# positive } \\
\text { Hl/total } \\
\text { \# at } \\
14 \mathrm{dpi}\end{array}$} \\
\hline & & & \multicolumn{3}{|c|}{ Tracheal } & \multicolumn{3}{|c|}{ Cloacal } & \\
\hline & & & $3 \mathrm{dpc}$ & $5 \mathrm{dpc}$ & $7 \mathrm{dpc}$ & $3 \mathrm{dpc}$ & $5 \mathrm{dpc}$ & $7 \mathrm{dpc}$ & \\
\hline 0 (Mock) & $0 / 8$ & 2 & $8 / 8(3.4 \pm 0.8)$ & $8 / 8(2.9 \pm 0.6)$ & $0 / 8$ & $2 / 8(3.7)$ & $5 / 8(3.4 \pm 0.2)$ & $5 / 8(3.2 \pm 0.5)$ & 8/8 (170) \\
\hline $10^{6}, 18$ & $1 / 4(3)$ & 2 & $3 / 4(3.3 \pm 1.0)$ & $3 / 4(2.9 \pm 0.9)$ & $0 / 4$ & $2 / 4(4.5 \pm 0.7)$ & $3 / 4(3.7 \pm 1.0)$ & $3 / 4(3.7 \pm 0.7)$ & 4/4 (320) \\
\hline $10^{6}, 19$ & 6/8 (13) & 2 & $2 / 8(3.5 \pm 0.7)$ & $1 / 8(2.3)$ & $0 / 8$ & $0 / 8$ & $0 / 8$ & $1 / 8(2.0)$ & $8 / 8(240)$ \\
\hline $10^{7}, 19$ & $3 / 5(5)$ & 2 & $1 / 5(2.7)$ & $0 / 5$ & $0 / 5$ & $0 / 5$ & $0 / 5$ & $1 / 5(2.3)$ & $5 / 5(272)$ \\
\hline 0 (Mock) & $0 / 7$ & 6 & $7 / 7(3.5 \pm 0.7)$ & $7 / 7(3.4 \pm 0.7)$ & $0 / 7$ & $3 / 7(3.9 \pm 0.5)$ & $5 / 7(3.7 \pm 1.0)$ & $5 / 7(3.3 \pm 0.8)$ & $7 / 7$ (525) \\
\hline $10^{6}, 18$ & $2 / 4(50)$ & 6 & $2 / 4(4.1 \pm 0.6)$ & $2 / 4(3.9 \pm 0.6)$ & $0 / 4$ & $1 / 4(3.5)$ & $2 / 4(4.3 \pm 0.4)$ & $2 / 4(3.6 \pm 0.1)$ & $4 / 4(360)$ \\
\hline $10^{6}, 19$ & $5 / 7(51)$ & 6 & $2 / 7(3.4 \pm 0.2)$ & $0 / 7$ & $0 / 7$ & 1/7 (3.7) & $1 / 7$ (3.5) & 1/7 (3.3) & $7 / 7$ (525) \\
\hline $10^{7}, 19$ & $4 / 5(64)$ & 6 & $1 / 5(3.7)$ & $1 / 5(3.7)$ & $0 / 5$ & $0 / 5$ & $0 / 5$ & $0 / 5$ & $5 / 5(640)$ \\
\hline
\end{tabular}


Table 5 Single-dose 2mH9N2:6WF10att in-ovo vaccination study in chickens challenged with low-pathogenic H9N2 (WF10) at 2 and 6 weeks post-hatching

\begin{tabular}{|c|c|c|c|c|c|c|}
\hline \multirow{3}{*}{$\begin{array}{c}\text { Vaccine dose } \\
\left(\text { EID }_{50}\right) / \text { embryo age (days) }\end{array}$} & \multirow{3}{*}{$\begin{array}{l}\text { \# positive } \mathrm{HI} / \text { total } \\
\text { \# before challenge }\end{array}$} & \multirow{3}{*}{$\begin{array}{l}\text { Age (in weeks) at } \\
\text { time of challenge }\end{array}$} & \multicolumn{4}{|c|}{ \# Shedding virus/total \# in swabs $\left(\log _{10} \mathrm{TCID}_{50} / \mathrm{ml} \pm \mathrm{SD}\right)$} \\
\hline & & & \multicolumn{3}{|c|}{ Tracheal } & \multirow[t]{2}{*}{ \# positive $\mathrm{HI} /$ total \# at $14 \mathrm{dp}$} \\
\hline & & & $3 \mathrm{dpc}$ & $5 \mathrm{dpc}$ & $7 \mathrm{dpc}$ & \\
\hline 0 (Mock) & $0 / 7$ & 2 & $7 / 7(2.7 \pm 0.6)$ & $7 / 7(2.5 \pm 0.3)$ & $0 / 7$ & $7 / 7$ (217) \\
\hline $10^{6}, 18$ & $3 / 5(14)$ & 2 & $2 / 5(2.2 \pm 0.2)$ & $2 / 5(2.3 \pm 0.4)$ & $0 / 5$ & $5 / 5(192)$ \\
\hline $10^{6}, 19$ & $6 / 7(32)$ & 2 & $1 / 7(2.5)$ & $0 / 7$ & $0 / 7$ & 7/7 (286) \\
\hline $10^{7}, 19$ & $3 / 4(32)$ & 2 & $1 / 4(2.3)$ & $0 / 4$ & $0 / 4$ & $4 / 4(260)$ \\
\hline 0 (Mock) & $0 / 7$ & 6 & $7 / 7(2.5 \pm 0.3)$ & $7 / 7(2.5 \pm 0.2)$ & $0 / 7$ & $7 / 7$ (320) \\
\hline $10^{6}, 18$ & $2 / 5(30)$ & 6 & $3 / 5(2.6 \pm 0.7)$ & $3 / 5(2.2 \pm 0.9)$ & $0 / 5$ & $5 / 5(224)$ \\
\hline $10^{6}, 19$ & $5 / 7(71)$ & 6 & $2 / 7(2.4 \pm 0.5)$ & $0 / 7$ & $0 / 7$ & 7/7 (446) \\
\hline $10^{7}, 19$ & $3 / 3(67)$ & 6 & $0 / 3$ & $0 / 3$ & $0 / 3$ & $3 / 3(227)$ \\
\hline
\end{tabular}

implicated in the cleavage of the HA of highly pathogenic avian influenza viruses - HPAIV [22]. The number of basic amino acid residues preceding the cleavage site determines recognition by either trypsin-like or furinlike proteases, with a string of basic amino acids allowing the latter to cause intracellular maturation of the $\mathrm{HA}$ at the level of the endoplasmic reticulum [23]. Furin-like protease cleavage produces mature virions that can spread cell to cell without having to reach the lumen of the respiratory or intestinal tracts. This permits the development of a fatal systemic infection, hence the so-called highly pathogenic influenza. Therefore, the cleavability of HA is one of the critical factors for viral tissue tropism and pathogenicity [24,25]. In this study, we modified the cleavage site of the influenza virus $\mathrm{H} 7$ and $\mathrm{H} 9 \mathrm{HA}$ protein genes to encode sequences corresponding to the H6 HA cleavage site ( $\mathrm{mH} 7$ and $\mathrm{mH} 9$ ) in order to improve hatchability after in ovo vaccination. It has been previously shown that the H6 HA cleavage site can transform a HPAIV of the H5N1 subtype into a LPAIV [19]. We have previously shown that a LPAI H5N1 virus carrying att mutations is amenable for in ovo vaccination resulting in $\geq 60 \%$ protection while maintaining at least $85 \%$ hatchability [2]. In this study we sought to examine whether the $\mathrm{mH} 7$ and $\mathrm{mH} 9$ att viruses viruses showed similar replication yields as unmodified $\mathrm{H} 7$ and $\mathrm{H} 9$ att viruses, and if these modified viruses were more amenable for in ovo vaccination without decreased immunogenicity. Growth kinetic studies in tissue culture cells showed similar yields for the $\mathrm{mH} 7$ compared to the unmodified $\mathrm{H} 7$ viruses (Figure 2) and similar results were obtained comparing the $\mathrm{mH} 9$ with the unmodified H9 pairs (not shown). As the safe "window" for in ovo vaccination of chicken embryos is between day 17 at 12-14 hours to day 19 at 2-4 hours [26], we chose days 18 and 19 for vaccination to test the effects on hatchability of the att vaccines. Hatchability studies clearly demonstrated that the $\mathrm{mH} 7$ and $\mathrm{mH} 9$ att viruses allowed for hatchability (90-93\%, 19-day old embryos) similar to the PBS inoculated controls (93-96\%), which were much higher than those obtained with the unmodified $\mathrm{H} 7$ or $\mathrm{H} 9$ att viruses (43-60\%, 19-day old embryos). We found that the combination of the modified HA cleavage site, vaccine dose, and time of vaccine delivery, had a significant impact on hatchability rates. Thus, 18-day old chicken embryos vaccinated with the $\mathrm{mH} 7$ or the $\mathrm{mH} 9$ att viruses showed improved hatchability rates compared to the unmodified HA att counterparts, but they were significantly lower than the rates obtained after vaccinating 19-day old embryos (Table 2). Likewise, increasing the dose to $10^{7} \mathrm{EID}_{50}$ of either $\mathrm{mH} 7$ or $\mathrm{mH} 9$ att viruses resulted in $10 \%$ hatchability loss compared to the same age embryos inoculated with $10^{6} \mathrm{EID}_{50}$ of the same viruses.

We speculate that the introduction of the alternative H6 HA cleavage site in the $\mathrm{mH} 7$ and $\mathrm{mH} 9$ att viruses (and perhaps in the $\Delta \mathrm{H} 5$ att virus) leads to reduced HA cleavage efficiency and, thus, these viruses exhibit growth restrictions at higher temperatures in vitro (Figure 3) and in vivo in 18-19-day old chicken embryos (Table 2). However, these viruses showed no defects in terms of virus yield at the permissive temperatures of 33 and $35^{\circ} \mathrm{C}$ in tissue culture (Figure 2) or in 10-day old chicken embryos. These characteristics are important because efficient immunogenicity was maintained without sacrificing virus yield. In fact, $2 \mathrm{mH} 7 \mathrm{N2}$ :6WF10att and 2mH9N2:6WF10att viruses can easily achieve titers on the order of $10^{8} \mathrm{EID}_{50} / \mathrm{ml}$ when grown in 10-day old embryonated chicken eggs (data not show), thus making them ideal for mass production.

In ovo vaccination is an attractive approach for vaccination of chickens, particularly broilers [26,27]. It helps to 'close the window' of susceptibility between vaccination and early exposure to infectious agents compared with post-hatch vaccination [27]. Because chickens already develop certain immunologic functions before 
hatching, in ovo vaccination stimulates both the innate and adaptive immune responses. Thus, in ovo vaccinated chicks develop an appreciable degree of protection by the time of hatching [27]. This indeed appears to be the case since in our approach chickens showed significant protection ( $\geq 70 \%$ ) when challenged as early as 2 weeks post-hatching. It is tempting to speculate that under industrial settings higher protection efficiencies could be obtained since automated systems would result in more accurate, controlled and efficient administration of the vaccine compared to our manual approach. In addition, because the $\mathrm{mH} 7$ and $\mathrm{mH} 9$ att viruses are more attenuated in vivo than the unmodified att counterparts, we further speculate that these HA genes are not likely to outcompete wild type influenza viruses through reassortment, and thus, should be safe to use in the field. The unprecedented spread of low pathogenic H7 and H9 influenza viruses in commercial settings, calls for the implementation of alternative prevention and control strategies. Our report provides for a viable alternative to the classical vaccination approaches against avian influenza.

\section{Acknowledgements}

We are indebted to Ivan Gomez and Yonas Araya for their assistance with the animal studies. We specially thank Andrea Ferrero for her laboratory managerial skills. We thank Robert Webster and Dennis Senne for providing the highly valuable virus strains. The opinions of this manuscript are those of the authors and do not necessarily represent the views of the granting agencies. This research was made possible through funding by NIAID-NIH grant (1U01A1070469-01), CSREES-USDA grant (2005-05523, 2006-01587, 2007-04981), and NIAID-NIH contract (HHSN266200700010C) and USDA-ARS. The funders had no role in study design, data collection and analysis, decision to publish, or preparation of the manuscript.

\section{Author details}

'Department of Veterinary Medicine, University of Maryland, College Park, 8075 Greenmead Drive, College Park, MD 20742-3711, USA. 'VirginiaMaryland Regional College of Veterinary Medicine, 8075 Greenmead Drive, College Park, MD 20742-3711, USA. 'Synbiotics Co. 8075 Greenmead Drive, College Park, MD 20742-3711, USA. ${ }^{4}$ Department of Animal and Avian Sciences, University of Maryland College Park, 1413 Animal Sciences Center, College Park, MD 20742-2311, USA.

\section{Authors' contributions \\ $Y C$ designed and performed reverse genetics virus rescue and in ovo vaccination studies and wrote the manuscript. HS perform molecular cloning, animal studies and co-wrote the manuscript. JY, HS, and RP designed and performed animal studies. TCS edited and proofread the manuscript. DRP was responsible for the overall study design, wrote, edited and proofread the manuscript. All authors read and approved the final manuscript.}

\section{Competing interests}

The authors declare that they have no competing interests.

Received: 12 October 2010 Accepted: 21 January 2011

Published: 21 January 2011

\section{References}

1. Swayne DE, Kapczynski D: Strategies and challenges for eliciting immunity against avian influenza virus in birds. Immunol Rev 2008, 225:314-331.
2. Song H, Nieto GR, Perez DR: A new generation of modified liveattenuated avian influenza viruses using a two-strategy combination as potential vaccine candidates. J Virol 2007, 81:9238-9248.

3. Wareing MD, Tannock GA: Live attenuated vaccines against influenza; an historical review. Vaccine 2001, 19:3320-3330.

4. Swayne $D E$, Beck JR, Kinney N: Failure of a recombinant fowl poxvirus vaccine containing an avian influenza hemagglutinin gene to provide consistent protection against influenza in chickens preimmunized with a fowl pox vaccine. Avian Dis 2000, 44:132-137.

5. Steel J, Lowen AC, Pena L, Angel M, Solorzano A, Albrecht R, Perez DR, Garcia-Sastre A, Palese P: Live attenuated influenza viruses containing NS1 truncations as vaccine candidates against $\mathrm{H} 5 \mathrm{~N} 1$ highly pathogenic avian influenza. J Virol 2009, 83:1742-1753.

6. Zhirnov OP, Klenk HD: Alterations in caspase cleavage motifs of NP and M2 proteins attenuate virulence of a highly pathogenic avian influenza virus. Virology 2009, 394:57-63.

7. Park MS, Steel J, Garcia-Sastre A, Swayne D, Palese P: Engineered viral vaccine constructs with dual specificity: avian influenza and Newcastle disease. Proc Natl Acad Sci USA 2006, 103:8203-8208.

8. Talon J, Salvatore M, O'Neill RE, Nakaya Y, Zheng H, Muster T, GarciaSastre A, Palese P: Influenza A and B viruses expressing altered NS1 proteins: A vaccine approach. Proc Natl Acad Sci USA 2000, 97:4309-4314.

9. Jin H, Lu B, Zhou H, Ma C, Zhao J, Yang CF, Kemble G, Greenberg H: Multiple amino acid residues confer temperature sensitivity to human influenza virus vaccine strains (FluMist) derived from cold-adapted A/Ann Arbor/6/60. Virology 2003, 306:18-24.

10. Steel J, Burmakina SV, Thomas C, Spackman E, Garcia-Sastre A, Swayne DE, Palese P: A combination in-ovo vaccine for avian influenza virus and Newcastle disease virus. Vaccine 2008, 26:522-531.

11. Toro H, Tang DC, Suarez DL, Sylte MJ, Pfeiffer J, Van Kampen KR: Protective avian influenza in ovo vaccination with non-replicating human adenovirus vector. Vaccine 2007, 25:2886-2891.

12. Hickman D, Hossain MJ, Song H, Araya Y, Solorzano A, Perez DR: An avian live attenuated master backbone for potential use in epidemic and pandemic influenza vaccines. J Gen Virol 2008, 89:2682-2690.

13. Tan J, Cooke J, Clarke N, Tannock GA: Molecular evaluation of responses to vaccination and challenge by Marek's disease viruses. Avian Pathol 2007, 36:351-359.

14. Sharma JM: Delayed replication of Marek's disease virus following in ovo inoculation during late stages of embryonal development. Avian Dis 1987, 31:570-576.

15. Ricks CA, Avakian A, Bryan T, Gildersleeve R, Haddad E, llich R, King S, Murray $L$, Phelps $P$, Poston $R$, et al: In ovo vaccination technology. Adv Vet Med 1999, 41:495-515.

16. Williams CJ, Zedek AS: Comparative field evaluations of in ovo applied technology. Poult Sci 89:189-193.

17. Reed $\mathrm{L}$, Muench $\mathrm{H}$ : A simple method for estimating 50 percent endpoints. Am J Hyg 1938, 37:493.

18. WHO Manual on Animal Influenza Diagnosis and Surveillance. [http://www. who.int/csr/resources/publications/influenza/whocdscsrncs20025rev.pdf].

19. Webby RJ, Perez DR, Coleman JS, Guan Y, Knight JH, Govorkova EA, McClain-Moss LR, Peiris JS, Rehg JE, Tuomanen El, Webster RG: Responsiveness to a pandemic alert: use of reverse genetics for rapid development of influenza vaccines. Lancet 2004, 363:1099-1103.

20. Alexander DJ: A review of avian influenza in different bird species. Vet Microbiol 2000, 74:3-13.

21. Alexander DJ: Avian influenza - diagnosis. Zoonoses Public Health 2008, 55:16-23.

22. Suarez DL: Avian influenza: our current understanding. Anim Health Res Rev 2010, 11:19-33.

23. Kido H, Okumura Y, Takahashi E, Pan HY, Wang S, Chida J, Le TQ, Yano M: Host envelope glycoprotein processing proteases are indispensable for entry into human cells by seasonal and highly pathogenic avian influenza viruses. J Mol Genet Med 2008, 3:167-175.

24. Lee CW, Lee YJ, Senne DA, Suarez DL: Pathogenic potential of North American $\mathrm{H} 7 \mathrm{~N} 2$ avian influenza virus: a mutagenesis study using reverse genetics. Virology 2006, 353:388-395.

25. Lee CW, Saif YM: Avian influenza virus. Comp Immunol Microbiol Infect Dis 2009, 32:301-310.

26. Williams CJ, Zedek AS: Comparative field evaluations of in ovo applied technology. Poult Sci 2010, 89:189-93. 
27. Negash T, al-Garib SO, Gruys E: Comparison of in ovo and post-hatch vaccination with particular reference to infectious bursal disease. A review. Vet Q 2004, 26:76-87.

doi:10.1186/1743-422X-8-31

Cite this article as: Cai et al:: Improved hatchability and efficient

protection after in ovo vaccination with live-attenuated H7N2 and H9N2

avian influenza viruses. Virology Journal 2011 8:31.

Submit your next manuscript to BioMed Central and take full advantage of:

- Convenient online submission

- Thorough peer review

- No space constraints or color figure charges

- Immediate publication on acceptance

- Inclusion in PubMed, CAS, Scopus and Google Scholar

- Research which is freely available for redistribution

Submit your manuscript at 